\title{
The moving bump in the light curves of SS For and RR Lyr
}

\author{
E. Guggenberger ${ }^{1}$, K. Kolenberg ${ }^{1,2}$ \\ ${ }^{1}$ Institut für Astronomie, Türkenschanzstrasse 17, 1180 Vienna, Austria \\ 2 Institute of Astronomy, University of Louvain, Celestijnenlaan 200B, B-3001 \\ Heverlee, Belgium
}

\begin{abstract}
High-precision multisite photometry was used to investigate the bump occurring before minimum light in the light curves of the two RRab Blazhko stars RR Lyr and SS For. For both stars the phase of the bump was found to be variable with a period equal to the Blazhko period. There seems to be a direct connection between the behavior of the bump and the Blazhko effect. As most models connect the phase of the bump to the stellar radius, a variable bump phase may provide constraints to the models for explaining the bump.
\end{abstract}

\section{Introduction}

In the majority of RR Lyrae stars a characteristic bump appears in the light curve just before luminosity minimum. Generally, shock or compression waves are thought to be responsible for this phenomenon, as RR Lyr stars don't meet the conditions for resonance that might cause the similar bump in cepheid light curves. Unlike the Hertzsprung progression in classical cepheids, no connection between the phase of the bump and the pulsation period of the star is known. Instead, we notice a strong change in the bump phase during the Blazhko cycle in SS For, one of our target stars observed in the framework of the Blazhko project (see http://www.univie.ac.at/tops/blazhko for project details). The Blazhko effect is a periodic change of pulsation amplitude and/or phase on time scales of usually tens of days (Blazhko 1907). With the luminosity maximum occuring at phase zero, the phase of the bump in SS For varies between 0.65 and 0.85 , which is, in other words, 20 per cent of the pulsation period. At the same time the strength of the bump is variable. The bump in RR Lyrae stars 
is generally believed to be the result of a shock wave, but there are different models to explain the phenomenon. The observed motion of the bump in the phase diagram might help to provide constraints for the models.

\section{Data and Methods}

For our investigation we combined data taken at the South African Astronomical Observatory (SAAO) and the Siding Spring Observatory in Australia (SSO) during a multisite campaign carried out from July through September 2005. SAAO data from the year 2004 as well as data gathered in 2000-2006 by the All Sky Automated Survey (ASAS, Pojmanski 2002) have also been added for analysis. ASAS data are in Johnson $V$ filter, SAAO and SSO are both in Johnson $V$ and $B$. A publication on the observations and data analysis is being prepared. The strong changes of the light curve shape around minimum light can be seen in Figure 1.

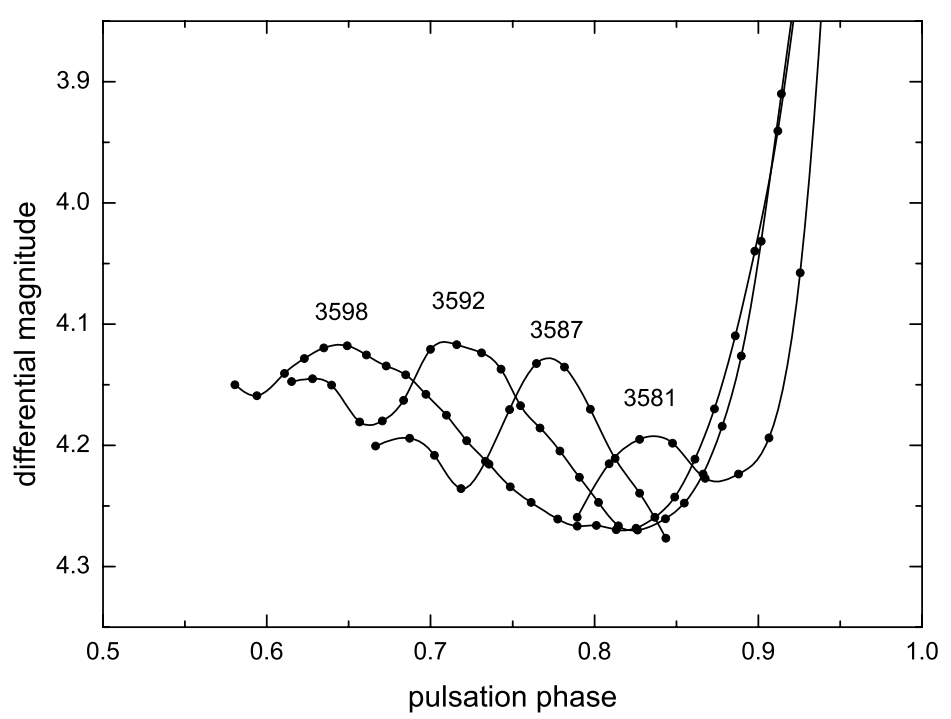

Figure 1: Phase diagram of SS For around bump phase for four different nights. Numbers in the figure represent Julian Date in the form $2450000+$. It can be seen how the bump changes in phase and amplitude during time intervals of 5 and 6 days, respectively. Scatter of data points is smaller than the symbols. 


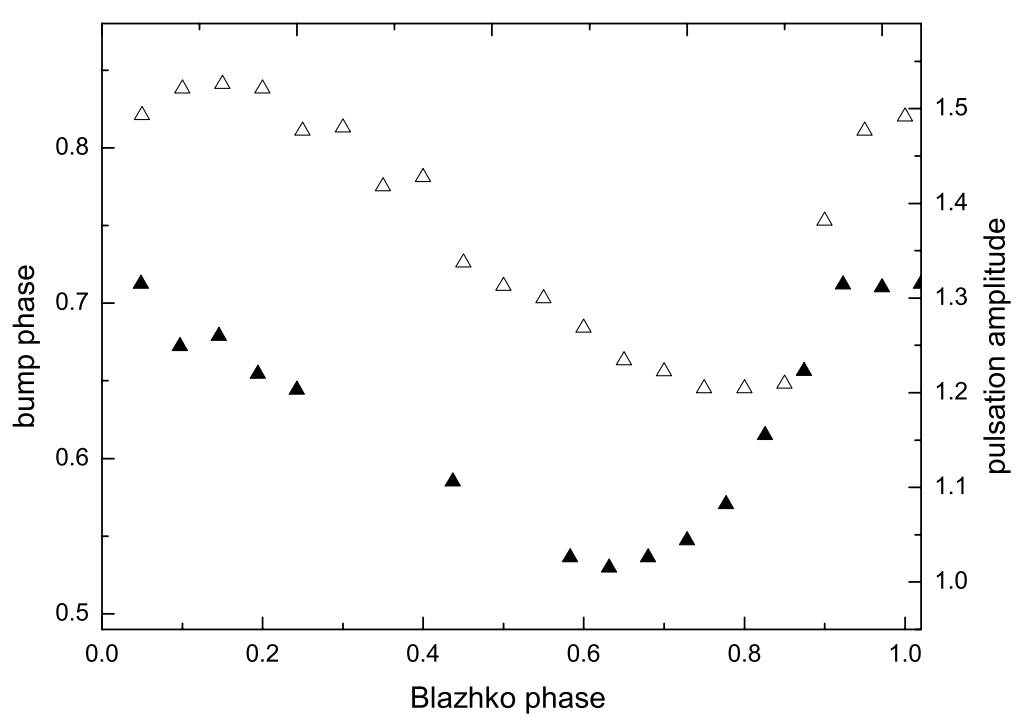

Figure 2: SS For: Change of pulsation amplitude (filled symbols) and phase of the bump maximum (open symbols) during a Blazhko cycle.

From this extensive data set we found a Blazhko period of about 34.7 days for SS For using Period04, a software package for statistical analysis of large astronomical data sets (Lenz \& Breger 2005). This period was used to calculate the Blazhko phase for all datapoints and to create phase bins. Using the $V$ data, 20 overlapping phase bins of 0.1 of the Blazhko period were created. The low scatter within the phase bins indicates that the correct value for the Blazhko period had been used. For each phase bin the pulsation phase of the bump maximum was measured and plotted against the Blazhko phase. The result can be seen in Figure 2.

We see that the phase of the bump maximum varies between a phase of 0.65 and 0.85 , and that the period of this variation is identical to the Blazhko period. Furthermore, it is obvious that the curve of the phase variation is nonsinusoidal, just like the variation of the pulsation amplitude during a Blazhko cycle. It is characterized by a slow progress to lower phases ('to the left') and a quicker motion to higher phases after the Blazhko minimum. The $\varepsilon$-value, which is as a measure of the skewness of a curve defined as

$$
\varepsilon=\phi_{\max }-\phi_{\min }
$$

is 0.35 both for the variation of the bump phase and the variation of the pulsation amplitude. 


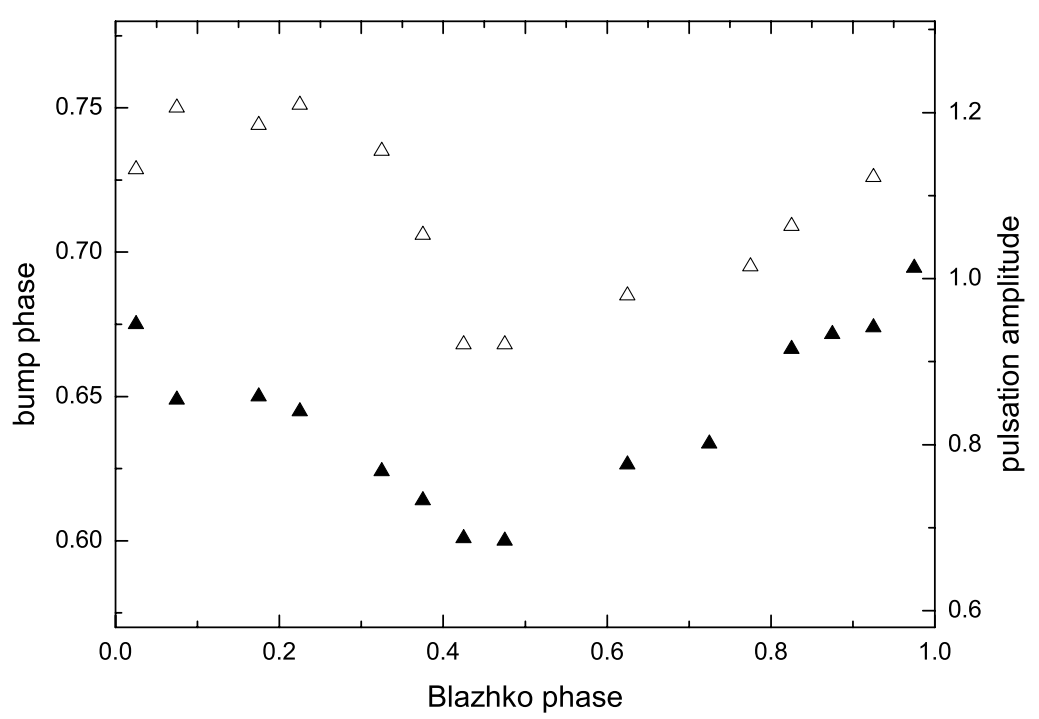

Figure 3: RR Lyr: Change of pulsation amplitude (filled symbols) and phase of the bump maximum (open symbols) during a Blazhko cycle.

The first question arising is whether this variability of bump phase is a common phenomenon among (Blazhko) RR Lyr stars. We performed the same test on a similar data set of RR Lyr. For details on this data set we refer to Kolenberg et al. (2006). In this case the Blazhko period found from the data set was 39 days. Again, the pulsation amplitude and the phase of the bump maximum were determined for each phase bin and plotted with respect to the Blazhko phase. The results turned out to be comparable (see Figure 3), altough the data of RR Lyr are of a lesser quality than those of SS For. Like for SS For, we see that the period of the bump motion is identical to the Blazhko period, and that the minimum bump phase is reached near Blazhko minimum, while the maximum bump phase is reached near maximum pulsation amplitude.

We see that the interval in phase in which the bump occurs is smaller for RR Lyr than it is for SS For. The lowest phase that is reached by the bump maximum in RR Lyr is 0.67 , the largest 0.75 with the total light curve maximum set to zero phase. This is a variation of only 8 per cent the pulsation period, compared to the high value of 20 per cent for SS For. The latter star is known for its rather large variations at minimum light (Lub 1977). In most Blazhko stars the variations around maximum light are most pronounced, while those around minimum light are much smaller. 


\section{Models}

RR Lyr stars are far from the condition of $P_{2} / P_{0}=0.5$ that could explain the existence of the bump in Cepheids by resonance. Therefore, shock waves are generally thought to be responsible for the observed bump in the light curves of RR Lyr stars. At present, there are two different models for explaining the bump, both involving shock waves: the 'echo' model and the 'infall' model (Gillet \& Crowe 1988). According to the infall model, fast inward moving upper atmospheric layers collide with deeper, slower ones during infall phase (Hill 1972). The echo model, on the other hand, connects the bump to a compression or shock wave that has been reflected on the stellar core.

\section{Discussion}

If the echo model is correct, one would expect a correlation between the stellar radius and the phase of the bump, as the shock wave travels a longer distance in a larger star than in a smaller one. This correlation was examined and confirmed by Carney et al. (1992) who calculated the elapsed time from the preceding minimum radius to the appearence of the bump for different stars and found a relation to the mean radius. This model can only explain a moving bump if the mean or equilibrium radius of the star changes. The infall model, on the other hand, connects the bump phase to the so-called expansion radius, which is given by Carney et al. (1992) as

$$
\Delta R=\int p\left(v_{\mathrm{rad}}-\gamma\right) d t,
$$

where $\mathrm{p}$ is the projection factor, $v_{\mathrm{rad}}$ is the radial velocity, and $\gamma$ the socalled $\gamma$-velocity during a Blazhko cycle. Though the Blazhko effect is not fully understood yet, the expansion radius is thought to be very likely to change during a Blazhko cycle. This would fit the variation of the phase of the bump as observed in our data. The bump appears late when the star is at Blazhko maximum and therefore is expected to have a larger expansion radius. The bump appears earlier around Blazhko minimum, when the star itself shows a variation that is less strong.

\section{Distinctiveness of the bump}

We also examined the strength of the bump both with respect to Blazhko phase and filter. The bump is stronger in the $B$ filter than it is in $V$, as it is clearly visible in a $B-V$ diagram (see Figure 4 ). We thus confirm the findings of previous authors such as Gillet \& Crowe (1988). 


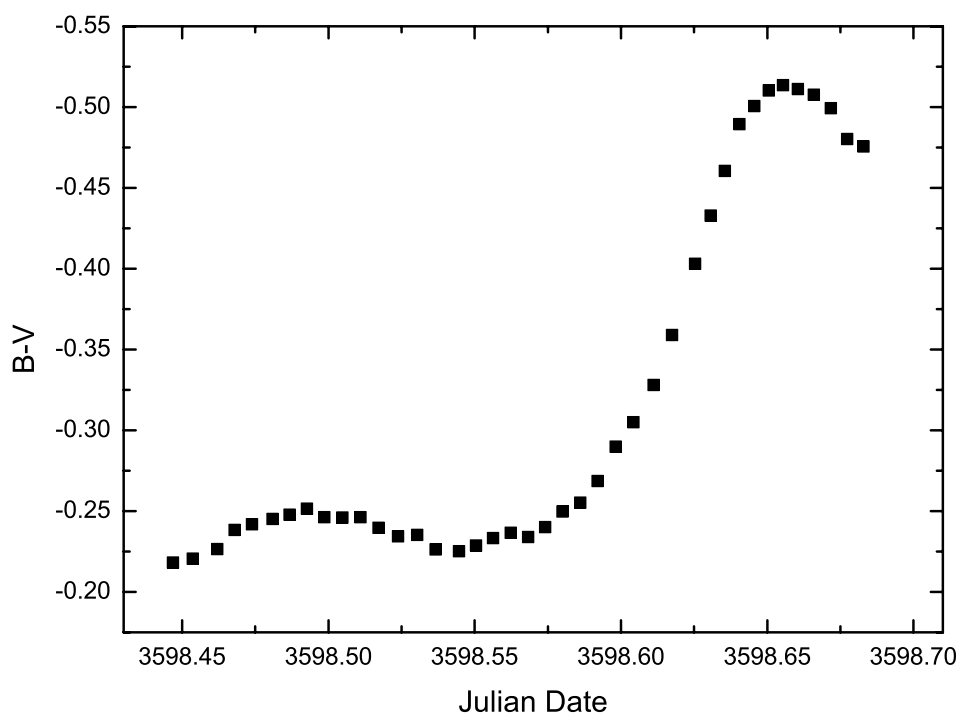

Figure 4: $B-V$ diagram of a selected night for SS For. Julian Date is given in the form $2450000+$. The bump is clearly visible and therefore stronger in Johnson $B$ than in $V$. Scatter is smaller than the symbols.

Regarding the Blazhko phase, however, we clearly see that in SS For the bump is most distinct during Blazhko minimum and almost vanishes around Blazhko maximum. For RR Lyr on the other hand previous authors such as Walraven (1949) and Fringant (1961) reported opposite behavior, finding the bump more conspicious during maximum amplitude than during minimum. We cannot confirm this as our investigation on the bump strength in RR Lyr does not allow us to conclude any noteworthy dependance on the Blazhko phase. Generally, one has to remark that it is very difficult to find a objective measure of the bump strengh as during its progress through the phase diagram it moves up and down the descending branch of the light curve and at the same time changes its width. In our analysis we used two different methods to determine the strength of the bump. First we simply measured the brightness of the bump maximum ignoring the motion along the descending branch. The second, more sophisticated method involved brightness measurements both left and right of the bump from which an average value was calculated. The bump amplitude then was defined as the difference between the bump maximum and this value. Both methods yielded comparable results. 


\section{Conclusions}

In in this paper we examined the behaviour of the bump in the light curve over the Blazhko cycle for two stars, SS For and RR Lyr. SS For is characterized by strong variations around minimum light. In both examined stars, the bump appearing just before minimum light moves back and forth in the phase diagram, with a period equal to the Blazhko period of the star. We conclude that there is a direct connection between the Blazhko effect and the motion of the bump. Models for the bump should therefore allow the bump phase to be variable. It will be necessary to investigate a larger sample of Blazhko and non-Blazhko stars to find out whether a variable bump is a common phenomenon.

Acknowledgments. The authors thank Michel Breger and the members of the TOPS team (Theory and Observation of Pulsating Stars) for fruitful discussions on the topic. Part of this investigation has been supported by the Austrian Fonds zur Förderung der wissenschaftlichen Forschung, project number P17097-N02.

\section{References}

Blazhko, S. 1907, Astr. Nachr., 175, 325

Carney, B.W., Storm, J., Trammell, S.R., \& Jones, R.V. 1992, PASP, 104, 44-56

Fringant, A. 1961, J.Obs., 44,165

Gillet, D., \& Crowe, R.A. 1988, A\&A, 199, 242-254

Hill, S.J. 1972, Astrophys. J., 178, 793

Kolenberg, K., Smith, H.A., Gazeas, K.D., et al. 2006, A\&A, in press

Lenz, P., \& Breger, M. 2005, CoAst, 146, 53

Lub, J. 1977, Astron. Astrophys. Suppl., 29, 345

Pojmanski, G. 2002, Acta Astronomica, 50, 177

Walraven, T. 1949, Bull. Astr.Inst., 11, 17 